\title{
Fabrication of High Aspect Ratio X-ray Grating Using X-ray Lithography*
}

\author{
Daiji NODA**, Hiroshi TSUJII**, Kazuma SHIMADA**, Wataru YASHIRO***, \\ Atsushi MOMOSE*** and Tadashi HATTORI** \\ **Laboratory of Advanced Science and Technology for Industry, University of Hyogo \\ 3-1-2 Koto, Kamigori, Ako, Hyogo 678-1205, Japan \\ E-mail:noda@lasti.u-hyogo.ac.jp \\ ${ }^{* * *}$ Department of Advanced Materials Science, School of Frontier Science, The University of Tokyo \\ 5-1-5 Kashiwanoha, Kashiwa, Chiba 277-8561 Japan
}

\begin{abstract}
X-ray radiographic imaging technique has found applications in various fields. However, it is not enough to get clear X-ray images of samples with low absorbance, such as biological soft tissues. To resolve this problem, we proposed a method using an X-ray Talbot interferometer of X-ray phase imaging. In this X-ray Talbot interferometer, X-ray gratings were required to have a fine, high accuracy, high aspect ratio structure. Then, we have developed and succeeded high aspect ratio X-ray gratings with a pitch of $5.3 \mu \mathrm{m}$ and a height of $30 \mu \mathrm{m}$ using X-ray lithography technique. We discuss that the X-ray gratings having a large effective area in order to obtain imaging size of practical use in medical application. In currently study, we have fabricated the X-ray gratings with a large effective area of $60 \mathrm{~mm} \times 60 \mathrm{~mm}$. And, we conducted X-ray phase tomography of mouse at the chest and abdominal using X-ray Talbot interferometer. As a result, we successfully observed soft tissues and high density tissues. With the aim of broadening a field of view, we try to fabricate X-ray gratings that have a pitch of below $5.3 \mu \mathrm{m}$ and larger area of $100 \mathrm{~mm}$ square. This result suggests that X-ray Talbot interferometer is a novel and simple method for phase sensitive X-ray radiography.
\end{abstract}

Key words: X-ray Grating, High Aspect Ratio Structure, X-ray Phase Imaging, X-ray Lithography, Electroplating

\section{Introduction}

Recently, X-ray imaging is a very important technology in the fields of medical, biological, material science, and inspection. However, it is not clear X-ray images of samples with low absorbance, such as biological soft tissues. The use of X-ray phase contrast provides a way to overcome this difficulty. Several methods for generating radiographic contrast from the information of X-ray phase shift have been investigated ${ }^{1}$. It is improvement in the sensitivity by about 1,000 times higher as compared with the conventional absorption imaging.

Numerous researches have studied a variety of methods of achieving phase imaging with X-ray gratings ${ }^{1-5}$. X-ray Talbot interferometer (XTI) ${ }^{1}$ is one of the methods. Figure 1 shows a setup of the XTI, and it employs two X-ray gratings and generates a contrast corresponding to the differential phase shift caused by a sample. It offers many advantages, such as very simple setup, needless of high mechanical stability, needless of high resolution in detector, wide area imaging, and broader energy band width.

Gold is a high X-ray absorptive material. However, in order to use 10-40 keV X-ray 


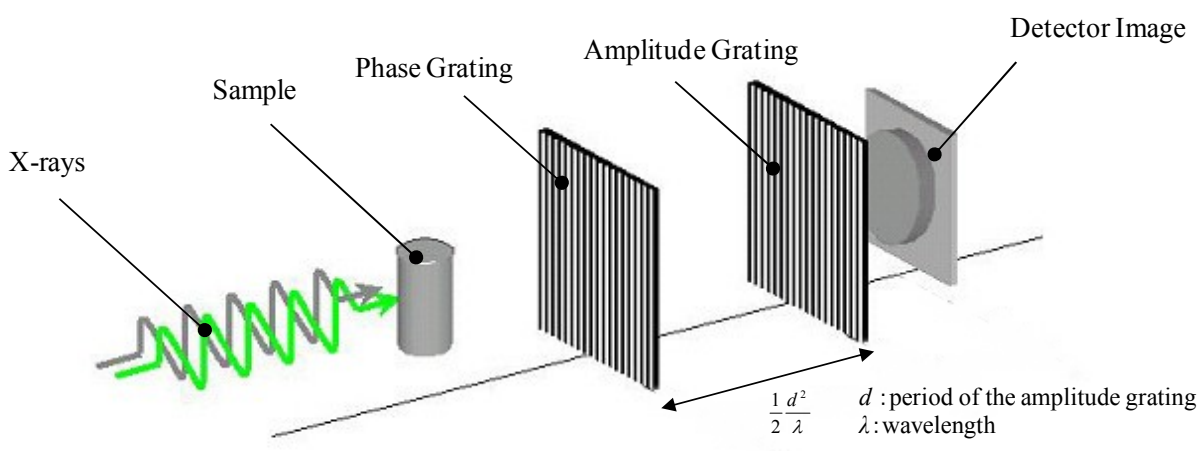

Figure 1. Configuration of an X-ray Talbot interferometer.

energies for XTI, gold thickness was required over $20 \mu \mathrm{m}$ height in amplitude X-ray grating because to block X-rays fully. The grating pitch must be shorter than X-ray coherence length. Therefore, it is difficult to make grating pattern structure with high aspect ratio. Then, the X-ray amplitude grating in the micrometer range pitch and high height $\mathrm{Au}$ structure could be developed ${ }^{6-8}$. We had first succeeded in fabricating with $20 \mathrm{~mm} \times 20 \mathrm{~mm}$ area and $8 \mu \mathrm{m}$ pitch structure by X-ray lithography and electroplating techniques ${ }^{7,8}$. Then, we obtained the phase tomography imaging ${ }^{3}$. In this paper, we discuss the X-ray grating having a pitch of $5.3 \mu \mathrm{m}$ and a large effective area of $60 \mathrm{~mm} \times 60 \mathrm{~mm}$ and more. The imaging area of XTI was depended on X-ray grating size. Therefore, larger size of X-ray grating was very useful to broaden of X-ray phase imaging in biological application.

\section{X-ray lithography technique}

For deep X-ray lithography, we used the NewSUBARU synchrotron radiation facility owned by our university. The beamline 2 (BL2) has a potential of large area patterning across up to A4-size area with a highly uniform pattern thickness. In operating energy at 1.0 $\mathrm{GeV}$, the BL2 has a spectrum range from 2 to $10 \mathrm{keV}$, with a peak of about $3.5 \mathrm{keV}^{9}$. Using this X-ray lithography technique, we could be obtained X-ray gratings pattern with line

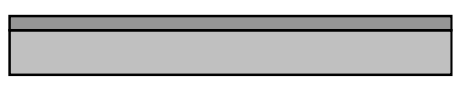

I. Seed layer deposition

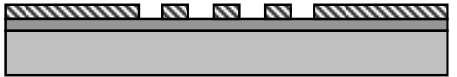

II. Resist patterning

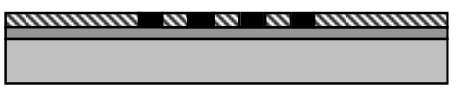

III. Electroplating of absorptive material

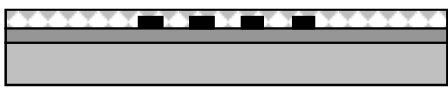

IV. Membrane coating

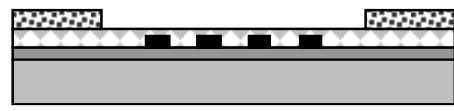

V.Frame bonding

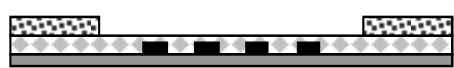

VI. Si wafer removing

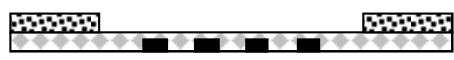

VII. Seed layer removing

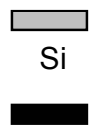

Ti

1010

resist

$\mathrm{Au}$ membrane
CFEOP

frame

Figure 2. Fabrication process of large area X-ray mask. 
width of several micrometers combined with a pattern thickness of over $20 \mu \mathrm{m}$ yields an aspect ratio of 10 or more ${ }^{7}$.

For large area X-ray lithography, we developed large area X-ray mask. Figure 2 shows the fabrication process of X-ray mask for grating patterns. Titanium layer was formed on $\mathrm{Si}$ substrate by sputtering to serve as electroplating of absorptive material (I). Next, negative photoresist of SU-8 was spin coating and patterning by ultraviolet (UV) lithography technique. Line and space patterns were created at a pitch of $5.3 \mu \mathrm{m}$ and a height of $4 \mu \mathrm{m}$ (II). Au was used as X-ray absorptive material, which was formed about $4 \mu \mathrm{m}$ height by electroplating between photoresist narrow lines (III), as shown in Fig. 3. Here, we used to form a resist of SU-8 as membrane because of large area X-ray mask. SU-8 was spin coating at $40 \mu \mathrm{m}$ thickness (IV). And, we attached a SUS frame in order to support the X-ray mask (V). Finally, Si substrate and Ti seed layer was removed by wet etching with potassium hydroxide $(\mathrm{KOH})$ and buffered hydrogen fluoride $(\mathrm{BHF})$, respectively (VI \& VII). Using these processes, the pitch pattern of $5.3 \mu \mathrm{m}$ X-ray mask was successfully fabricated with a large effective area of $60 \mathrm{~mm} \times 60 \mathrm{~mm}$ as shown in Fig. 4 .

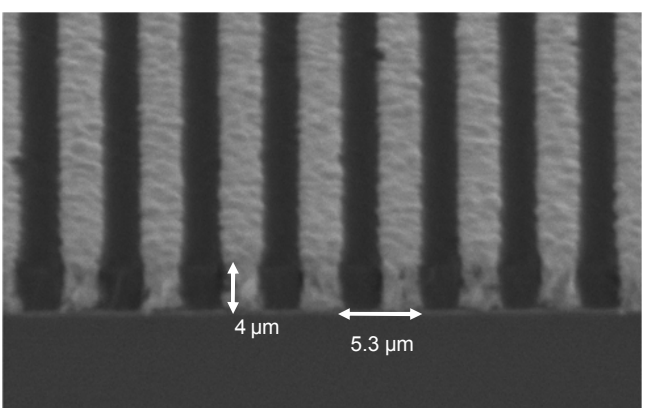

Figure 3. SEM image of $\mathrm{Au}$ absorber in cross section.

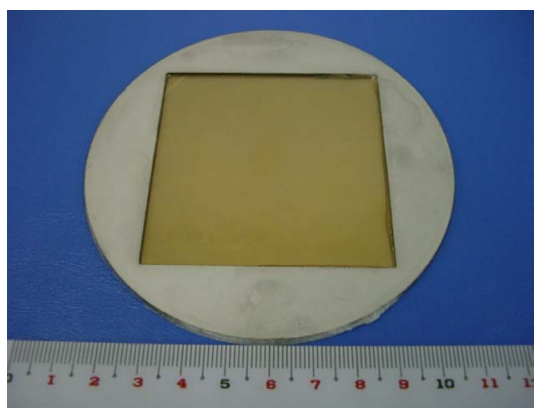

Figure 4. External view of X-ray mask with $60 \mathrm{~mm} \times 60 \mathrm{~mm}$.

\section{Fabrication of high aspect ratio gratings}

For the deep X-ray lithography, we used a negative type photoresist SU-8. Although SU-8 was originally designed for UV lithography, it could also be used in deep X-ray lithography of high accuracy, high aspect ratio pattern. To deposit Au as an X-ray absorber of X-ray mask, we decided on a target $\mathrm{Au}$ thickness of $30 \mu \mathrm{m}$ using electroplating. Accordingly, we have obtained the photoresist thickness at $35 \mu \mathrm{m}$.

If a structure with a large area and a narrow pitch was X-ray exposed, sticking was observed in many places probably because surface tension occurred drying of the developed and rinsed in washing process, as shown in Fig. 5. Then, in order to solve this problem, we used supercritical washing and dry machine (product by RyuSyo Industrial Co., Ltd.) using carbon dioxide $\left(\mathrm{CO}_{2}\right)$ as the supercritical fluid ${ }^{10}$ because it is safe and have a low critical point lower than those of other fluids. It can be dried without surface tension because a supercritical fluid behaves as a no surface gas. Figure 6 shows scanning electron microscopy (SEM) image of photoresist structure with high aspect ratio without sticking. From this figure, processing depth was obtained about $35 \mu \mathrm{m}$ with narrow pitch of $5.3 \mu \mathrm{m}$. Thus, an aspect ratio of resist structure was achieved about 13 .

$\mathrm{Au}$ designed to form the absorbing part for the X-ray grating was formed by electroplating. In this electroplating, chromium was used as seed layer. We used a non-cyanic based solution for Au electroplating. With the resist structure has an aspect ratio as higher as 10, pretreatment using a moistening agent was used to speed up the circulation of $\mathrm{Au}$ electroplating bath. And, we have developed uniformity electroplating method for 
large effective are of X-ray grating. Figure 7 shows the picture of large area X-ray grating after $\mathrm{Au}$ electroplating as large effective area of $60 \mathrm{~mm} \times 60 \mathrm{~mm}$. SEM image of X-ray grating in cross section with a narrow pitch of $5.3 \mu \mathrm{m}$ and a height of about $30 \mu \mathrm{m}$ are realized as shown in Fig. 8.

With the aim of broadening a field of view, we try to fabricate X-ray gratings that have a pitch of below $5.3 \mu \mathrm{m}$ and larger area of $100 \mathrm{~mm}$ square. Figure 9 shows the picture of

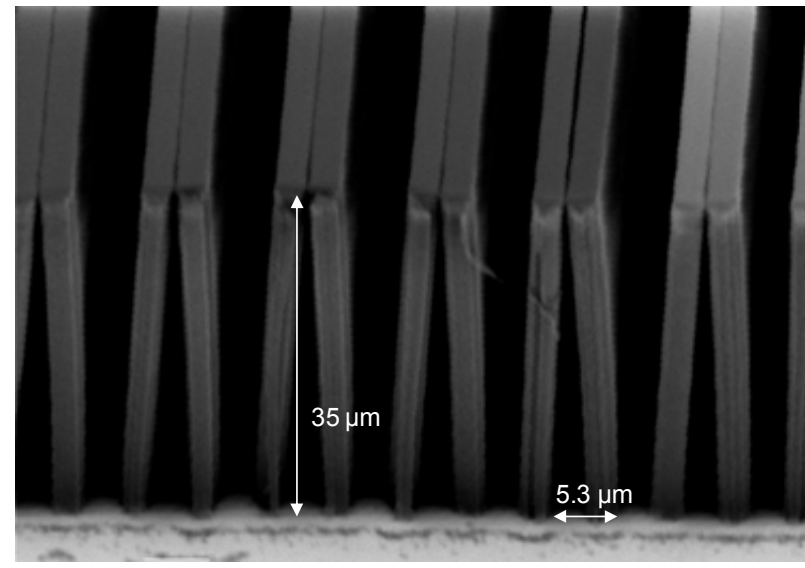

Figure 5. SEM image of narrow pitch resist structure in cross section with sticking.

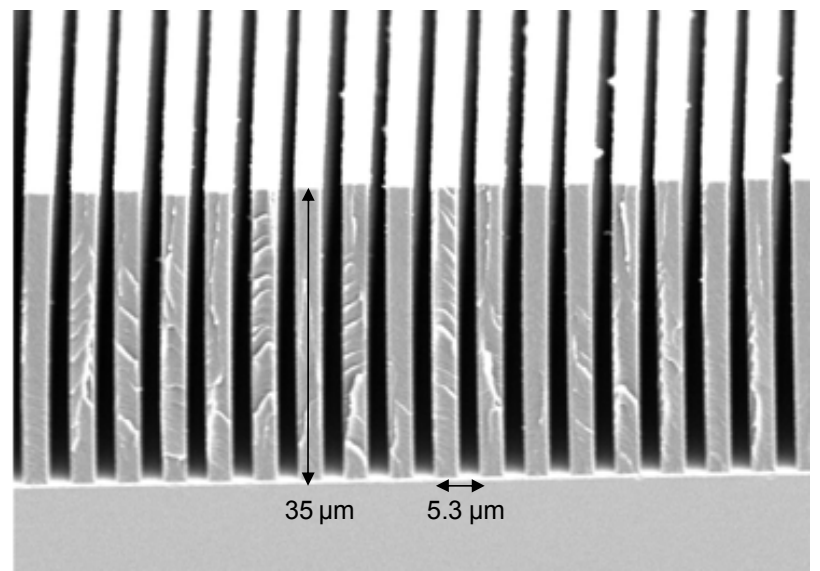

Figure 6. SEM image of narrow pitch structure in cross section without sticking.

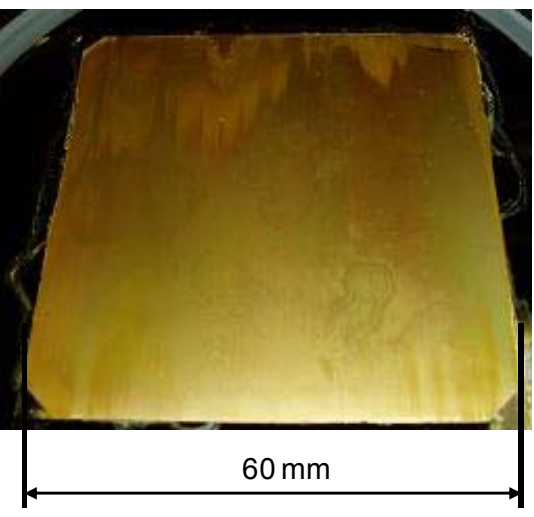

Figure 7. External view of $\mathrm{X}$-ray grating with effective area of $60 \mathrm{~mm} \times 60$ $\mathrm{mm}$ and a pitch of $5.3 \mu \mathrm{m}$.

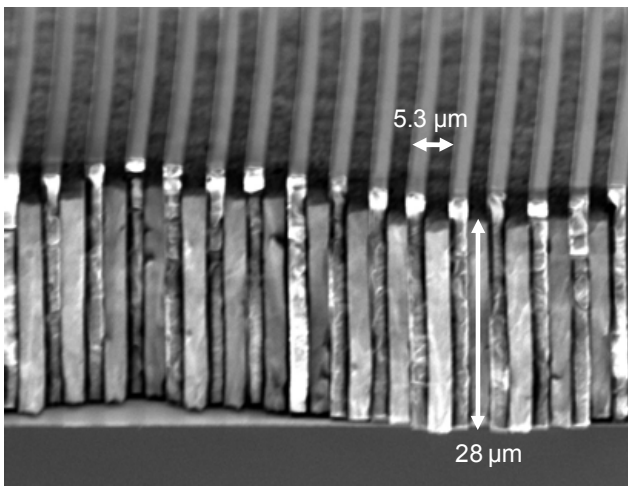

Figure 8. SEM image of $\mathrm{X}$-ray grating in cross section with a pitch of $5.3 \mu \mathrm{m}$ and an $\mathrm{Au}$ height of $28 \mu \mathrm{m}$. 


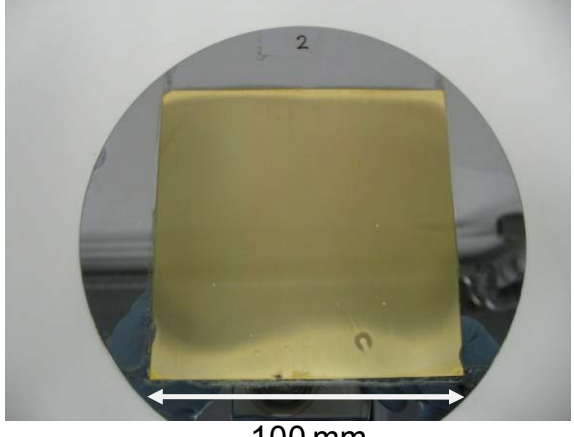

$100 \mathrm{~mm}$

Figure 9. External view of X-ray grating with effective area of $100 \mathrm{~mm} \times 100$ $\mathrm{mm}$ and a pitch of $5.3 \mu \mathrm{m}$.

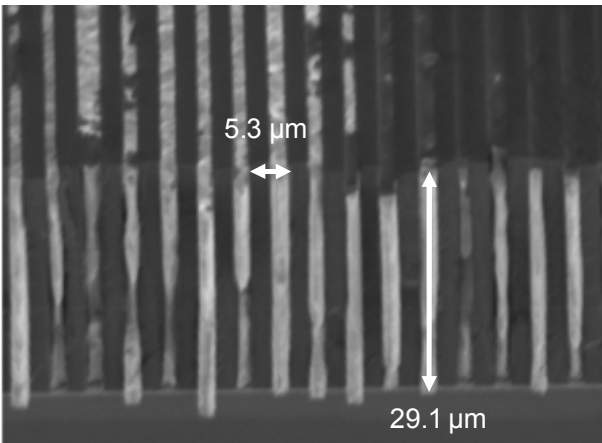

Figure 10. SEM image of X-ray grating in cross section with a pitch of $5.3 \mu \mathrm{m}$ and an $\mathrm{Au}$ height of $29 \mu \mathrm{m}$.

large area X-ray grating after Au electroplating as large effective area of $100 \mathrm{~mm} \times 100 \mathrm{~mm}$ with a same pitch of $5.3 \mu \mathrm{m}$ and a height of about $30 \mu \mathrm{m}$. SEM image of X-ray grating in cross section with a narrow pitch of $5.3 \mu \mathrm{m}$ and a height of about $30 \mu \mathrm{m}$ are realized as shown in Fig. 10.

The phase grating was also fabricated in a similar process, expect that X-ray lithography was used, and had a gold pattern.

\section{Evaluation of X-ray grating}

We experimented and evaluated the X-ray amplitude gratings using a compact laboratory X-ray source in The University of Tokyo and the BL14C1 in Photon Factory of High Energy Accelerator Research Organization (KEK-PF). We decided to measure the visibility of moiré pattern ${ }^{3}$ obtained from the image detector with no sample placed in front of XTI. The higher visibility value, the higher imaging is obtained sensitivity. Although the visibility value decreased with increasing X-ray energy, the X-ray Talbot interferometer exhibited moiré fringes with a visibility sufficient for phase imaging at $0.04 \mathrm{~nm}$. Figure 11 shows a visibility map of X-ray grating with effective area of $60 \mathrm{~mm} \times 60 \mathrm{~mm}$. In this case, we used micro focus X-ray source at a wavelength of $0.05 \mathrm{~nm}(25.5 \mathrm{keV})$ for evaluation. As a result, we successfully verified the Talbot effect, obtaining 29-35\% in all effective area.

Figure 12 shows a visibility map of X-ray grating with effective area of $100 \mathrm{~mm} \times 100$ $\mathrm{mm}$. In this case, we used the synchrotron radiation of KEK-PF at a wavelength of $0.07 \mathrm{~nm}$ $(17.7 \mathrm{keV})$ for evaluation. As a result, we successfully verified the Talbot effect, obtaining about $70 \%$ in all effective area. These results are very good value. Therefore, the X-ray gratings could be used for X-ray phase imaging in XTI.

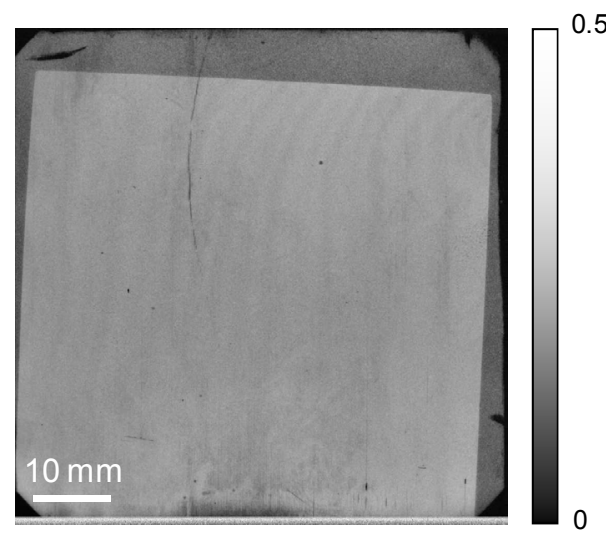

Figure 11. Visibility map of moiré pattern with effective X-ray grating area of $60 \mathrm{~mm} \times 60 \mathrm{~mm}$. 


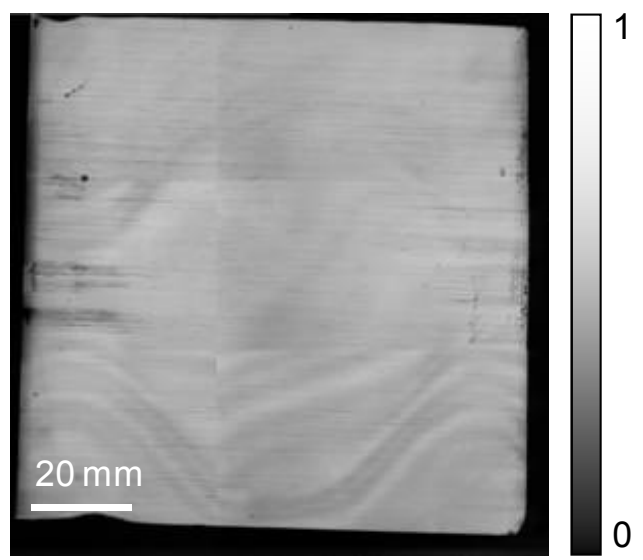

Figure 12 . Visibility map of moiré pattern with effective X-ray grating area of $100 \mathrm{~mm} \times 100 \mathrm{~mm}$.

\section{Phase tomography}

We have obtained a result of phase tomography performed for a one-week-old mouse. An experiment was carried out at BL20B2 of SPring-8, Japan with $24.8 \mathrm{keV} \mathrm{X-rays} \mathrm{from} \mathrm{a}$ bending section. The sample was put in a plastic tube $2 \mathrm{~cm}$ in diameter filled with formalin. Because of the problem of bubble generation in the cell due to intense X-ray irradiation, beam intensity was moderately reduced by undulator-gap detuning. X-ray images were recorded with a CCD camera with a phosphor screen coupled with a lens. The measurement by a five-step fringe scan was repeated at each angular position of the sample rotation with a step of $0.72^{\circ}$ over $180^{\circ}$. In this study, we used amplitude X-ray grating with a large effective area of $60 \mathrm{~mm} \times 60 \mathrm{~mm}$, as shown in Fig. 7. Figure 13 shows preliminarily reconstructed phase tomography at the chest and abdominal regions ${ }^{11}$. It is well known that phase contrast approach is potentially a thousand times more sensitive than absorption contrast approach according the cross-section ratio between the phase shift and absorption. These demonstrations of the XTI are a promising method for many applications of X-ray imaging. And, a phase imaging of XTI using a large effective area X-ray grating of $100 \mathrm{~mm}$ $\times 100 \mathrm{~mm}$ is expected in many fields such as medical applications.

\section{Conclusions}

We have fabricated X-ray mask and X-ray gratings having a pitch of $5.3 \mu \mathrm{m}$ and a large effective area of $60 \mathrm{~mm} \times 60 \mathrm{~mm}$ for X-ray Talbot interferometer for use in X-ray phase tomography imaging. To make high aspect ratio $\mathrm{X}$-ray grating, we developed the fabrication techniques composed of MEMS technology, X-ray lithography, and narrow pitch electroplating. When the large area and narrow pitch structure of X-ray grating was exposed by synchrotron radiation, sticking was observed in many places because of surface tension. Then, we introduced a supercritical washing and drying machine using $\mathrm{CO}_{2}$ as the supercritical fluid. Through use of supercritical washing and drying machine, we obtained the X-ray grating without surface tension. After Au electroplating, a large area X-ray grating could be fabricated. Then, we succeeded X-ray grating of Au absorber structure with a pitch of $5.3 \mu \mathrm{m}$, a height of about $30 \mu \mathrm{m}$, and an aspect ratio of over 10 . With the aim of broadening a field of view, we could be fabricated X-ray grating that have large effective area of $100 \mathrm{~mm} \times 100 \mathrm{~mm}$.

We decided to measure the visibility of moiré pattern. These results are obtained very good value. Therefore, the X-ray gratings could be used for X-ray phase imaging in XTI. We conducted X-ray phase tomography of mouse filled with formalin at the chest and 


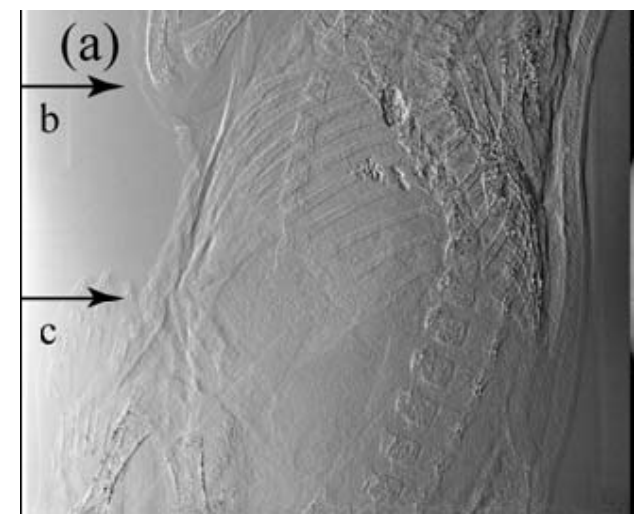

(a) Projection image
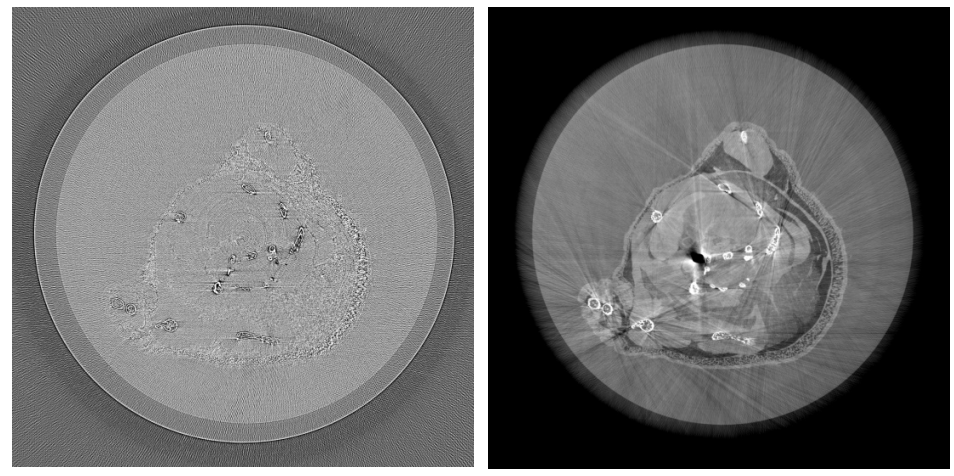

(b) X-ray tomography image at the chest in phase (right image ) and absorption (left imge) contrast.
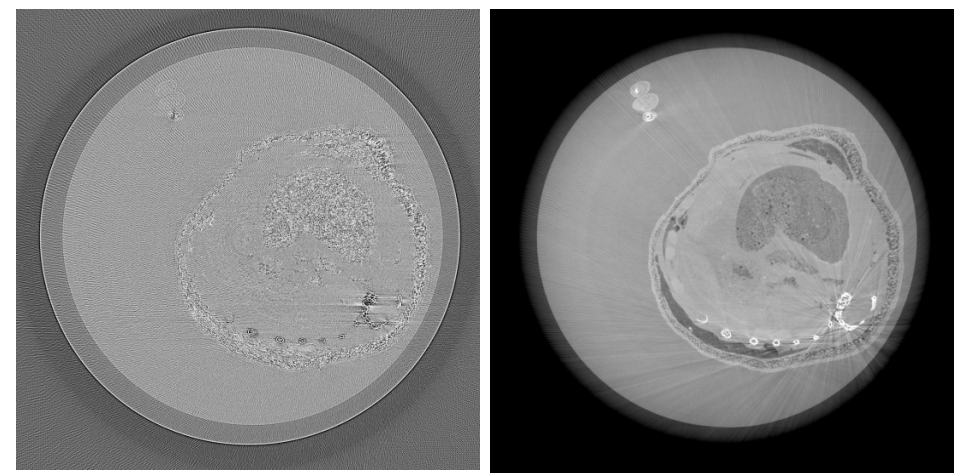

(c) X-ray tomography image at the abdominal region in phase (right image ) and absorption (left imge) contrast

Figure 13. X-ray tomography image of mouse.

abdominal regions. As this result, we successfully observed soft tissues and high density tissues more sensitive than absorption contrast. These demonstrations of the XTI are a promising method for many applications of X-ray imaging.

This technique would be useful as a means of fabricating X-ray gratings for use in $\mathrm{X}$-ray phase imaging as $\mathrm{X}$-ray Talbot interferometer, and, in addition, it would serve as a technique for fabricating other devices requiring a high aspect ratio micro-structure.

\section{Acknowledgment}

This research was supported by the research project "Development of Systems and Technology for Advanced Measurement and Analysis" from the Japan Science and Technology Agency (JST). 


\section{References}

1. A. Momose, S. Kawamoto, I. Koyama, Y. Hamaishi, K. Takai and Y. Suzuki, "Demonstration of X-ray Talbot interferometry", Japanese Journal of Applied Physics, Vol. 42, No. 7B, 2003, pp. L866-L868.

2. A. Momose, "Recent advances in X-ray phase imaging", Japanese Journal of Applied Physics, Vol. 44, No. 9A, 2005, pp. 6355-6367.

3. A. Momose, W. Yashiro, Y. Takeda, Y. Suzuki and T. Hattori, "Phase tomography by X-ray Talbot interferometry for biological imaging", Japanese Journal of Applied Physics, Vol. 45, No. 6A, 2006, pp. 5254-5262.

4. C. David, B. Nöhammer, H. H. Solak and E. Ziegler, "Differential X-ray phase contrast imaging using a shearing interferometer", Applied Physics Letters, Vol. 81, 2002, pp. 3287-3289.

5. F. Pfeiffer, T. Weitkamp, O. Bunk and C. David, "Phase retrieval and differential phase-contrast imaging with low- brilliance X-ray sources", Nature Physics, Vol. 2, 2006, pp. 258-261.

6. C. David, J. Bruder, T. Rohbeck, C. Grünzweig, Co. Kottler, A. Diaz, O. Bunk and F. Pfeiffer, "Fabrication of diffraction gratings for hard X-ray phase contrast imaging", Microelectronic Engineering, Vol. 84, 2007, pp. 1172-1177.

7. D. Noda, M. Tanaka, K. Shimada and T. Hattori, "Fabrication of diffraction grating with high aspect ratio using X-ray lithography technique for X-ray phase imaging", Japanese Journal of Applied Physics, Vol. 46, No. 2, 2007, pp. 849-851.

8. M. Matsumoto, K. Takiguchi, M. Tanaka, Y. Hunabiki, H. Takeda, A. Momose, Y. Utsumi and T. Hattori, "Fabrication of diffraction grating for X-ray Talbot interferometer", Microsystem Technologies, Vol. 13, 2007, pp. 543-546.

9. Y. Utsumi, T. Kishimoto, T. Hattori and H. Hara, "Large area and wide dimensions X-ray lithography using energy variable synchrotron radiation", Microsystem Technologies, Vol. 13, 2007, pp. 417-423.

10. M. Tanaka, Y. Takeda, D. Noda, W. Yashiro, K. Okuda, A. Momose and T. Hattori, "Fabrication of gratings for an X-ray Talbot interferometer", Proceedings of the 2006 IEEE International Symposium on Micro-Nano Mechatronics and Human Science, No. P1-32 (2006-11), pp. 491-496.

11. A. Momose, Y. Takeda, M. Shinohara, K. Wan, W. Yashiro, D. Noda and T. Hattori, "Full-body phase tomography of a mouse by X-ray Talbot interferometry", Abstracts of the 9th International Conference on Biology and Synchrotron Radiation, 2007-8, pp. 54. 